\title{
Volumetric De-Escalation and Improved Acute Toxicity with Proton Craniospinal Irradiation Using a Vertebral Body Sparing Technique
}

\author{
Brian Chou ${ }^{1}$, Austin Hopper ${ }^{2}$, Jennifer Elster ${ }^{3}$, John Crawford ${ }^{3}$, Kristen McConnell ${ }^{2}$, \\ Andrew Chang ${ }^{4}$, Arno Mundt ${ }^{2}$, and Iain MacEwan ${ }^{2}$ \\ ${ }^{1}$ Loyola University Health System \\ ${ }^{2}$ University of California San Diego \\ ${ }^{3}$ Rady Children's Hospital San Diego \\ ${ }^{4}$ California Protons Cancer Therapy Center
}

April 27, 2021

\begin{abstract}
Purpose: Craniospinal irradiation (CSI) has historically treated the entire vertebral body (VB) in growing children. Vertebral body sparing proton craniospinal irradiation (VBSpCSI) is a technique which spares the majority of the VB from significant irradiation. This retrospective study reviews the acute toxicity of VBSpCSI compared to photon CSI. Methods: Pediatric CSI patients treated between 2008 and 2018 were evaluated. Patients were stratified to the VBSpCSI cohort or the photon cohort and analyzed for acute toxicity profile during treatment and disease-free survival (DFS). Statistical analysis was performed using Kaplan-Meier log rank analysis for DFS and Fisher's exact test for toxicity. Results: Twenty-five patients received VBSpCSI and 13 patients received photon CSI. Mean patient age at treatment was $7.5 \mathrm{y}$ (range 2 to 16). The cohorts were well-matched with respect to gender, age, and CSI dose. Two-year DFS was similar between cohorts ( $81 \%$ VBSpCSI vs $61 \%$ photon, $\mathrm{p}=0.18)$. Patients receiving VBSpCSI had lower rates of grade $2+$ GI toxicity $(24 \%$ vs $76.5 \%$, p=0.005), grade $2+$ nausea $(24 \%$ vs $61.5 \%, \mathrm{p}=0.035)$, and any-grade esophagitis $(0 \%$ vs $38 \%, \mathrm{p}=0.0026)$. Patients treated with VBSpCSI had lower red blood cell transfusion rates $(21.7 \%$ vs $60 \%, \mathrm{p}=0.049)$ and grade $4+$ lymphopenia $(33.3 \%$ vs $77.8 \%, \mathrm{p}=0.046)$. Conclusions: VBSpCSI in children is a volumetric de-escalation from traditional volumes which irradiate the entire vertebral body. Based on our results, VBSpCSI was associated with less acute gastrointestinal and hematologic toxicity. The study adds to the growing body of evidence supporting the use of protons over photons for pediatric CSI.
\end{abstract}

\section{Hosted file}

Manuscript.pdf available at https://authorea.com/users/410404/articles/519791-volumetricde-escalation-and-improved-acute-toxicity-with-proton-craniospinal-irradiation-using-avertebral-body-sparing-technique

\section{Hosted file}

Tables.pdf available at https://authorea.com/users/410404/articles/519791-volumetric-deescalation-and-improved-acute-toxicity-with-proton-craniospinal-irradiation-using-avertebral-body-sparing-technique 